\title{
The Role of Artificial Intelligence in Human Resource Management
}

\author{
Dr. S. Tephillah Vasantham \\ Assistant Professor, Department of Business Administration, The American College, Madurai, Tamil Nadu, India \\ E mail: tephillah88@gmail.com
}

\begin{abstract}
This paper deals with the Role of Artificial Intelligence (AI) in Human Resource Management (HRM). We can see in the present globalized world, the customary methods of how business is directed are being tested. There could be not, at this point just nearby firms as contenders, yet associations need to contend continually on a worldwide level as innovation is making the world more modest. This infers that for an association to keep awake to date and maintain an upper hand and accepting these new mechanical advancements is critical. HRM includes a wide range of viewpoints, like preparing workers, enrollment, representative relations, and the advancement of the association. People fill in as a wellspring of information and ability which each association can and should draw on. Hence, obtaining and holding these kinds of workers through enrollment assume a major part today. Because of the significance Human Resource (HR) has for the association, the enrollment interaction by which all this asset is acquired is the way to progress. The enlistment cycle used to be longer and take a lot of time and suggest a lot of administrative works for the spotters, anyway this has as of now gradually began to change with online enrollment getting normal. This paper deals with the various applications and the advantages of implementing Artificial Intelligence in Human Resource management.
\end{abstract}

Keywords — Artificial Intelligence; Human Resource Management; Innovation; Human Intelligence.

\section{Introduction}

In later years because of the innovative changes, research has been directed on how these two important parts of HRM and innovation could be joined. Normally, contemplates are directed at how the enrollment interaction can be smoother and enhanced with the assistance of innovation (Galanaki, Lazazzara and Parry, 2019). At this moment, the spotlight lies significantly more on mechanical advances helping spotters, for a model the interaction is getting more computerized. Because of this, it very well may be expressed that the human touch in enrollment is getting reduced (Bondarouk and Brewster, 2016). In the article by Baxter (2018) he attempts to anticipate the patterns which will assume control over enrollment in 2019. He recommends prescient investigation to remove some portion of all the speculating which happens in enlistment, however, he likewise raises AI as a device that will be utilized when talking up-and-comers (Baxter, 2018). This proposition expects to investigate the part of one of the fresher innovations: Artificial Intelligence (AI). The use of AI to HRM was perhaps the most amazing pattern among enrollment experts in 2018. The selection of AI in HRM and in enlisting can be called as 'the new time of HR', since AI changes the enrollment business by supplanting routine assignments that have been led by human spotters (Upadhyay and Khandelwal, 2018).

Tecuci (2012) makes reference to that AI as a field is wide and a multidisciplinary space, which can be misused in registering disciplines as well as in semantics and theory. Simulated intelligence can take various structures, like robots, bots or programming (Tecuci, 2012). The idea of $\mathrm{AI}$ is quite possibly the most novel spaces in designing and science and it has been concentrated since the Second World War. The name of Artificial Intelligence was confirmed in 1956 (Stuart and Norvig, 2016). Salin and Winston (1992) characterized AI just like a bunch of strategies that permit PCs to achieve assignments that would some way or another require the thinking abilities that human intelligence brings. As per Nilsson (2005) machines ought to have the option to do the vast majority of the positions that human intelligence request, which he calls for human-level AI. This proposal won't zero in on AI as far as genuine robots, but instead on the execution of AI in various programming which organizations use inside the enlistment cycle.

\section{Human Resource Management}

There are numerous meanings of human asset the board presented by a scope of analysts, anyway the vast majority of the definitions do supplement one another. A definition by Schemerhorn (2001) is that HRM is the way you can acquire and build up a labor force which is gifted, to help the organization accomplishes its objectives, just as its central goal, vision and various destinations within reach. Another definition is that HRM is a way to deal with representative administration determined to hold a labor force which is both fit and submitted by various methods, for example, social, primary and faculty to bring the association an upper hand (Story, 2004). With the end goal of this investigation, HRM will be characterized as the way toward getting and keeping up new abilities, capacities and skills in an association through its labor force by the methods for various administration strategies.

HRM rehearses incorporate selecting new representatives, overseeing workers, recruiting workers and 
improvements (Wall and Wood, 2005). The greater parts of these practices have a particular spotlight on holding new representatives and keeping up their acceptable level. This is on the grounds that HR are a particularly unique piece of the organization and is truly changing, thusly it needs the correct administration by an association (Bibi, Pangil and Johari, 2016).

The administration and maintenance of HRM can be contended to have a unique significance inside assembling organizations which sees an emphasis on advancement inside assembling to get a relative benefit and better exhibitions (Youndt, Snell, Dean and Lepak, 1996). The job that HRM have inside an association have changed seriously during numerous years and are not, at this point just utilized as an approach to deal with an association's inner expenses of work (Becker and Gerhart, 1996). Later investigates are investigating HRM similar to an essential resource for associations where workers are the key resources and how to obtain and deal with these assume the main part (Bas, 2012). In the accompanying segment enrollment in HRM will be talked about followed by a part on determination in HRM.

\section{Enlistment in HRM}

The exploration led inside enrollment as a piece of HRM has expanded in the later many years and there is currently more accessible examination on what enlistment really mean for candidate practices and worker conduct (Taylor and Collins, 2000). Enlistment is characterized as the act of tracking down the correct applicants which make up a competitor pool which fits an open occupation opportunity that an organization has (Stoilkovska, Ilieva and Gjakovski, 2015). Enlistment can likewise be supposed to be the focal point inside HRM, as it is those workers that are employed who will be subject later on to the next HRM rehearses (Griepentrog, Harold, Holtz, Klimoski and Marsh, 2012). This is additionally upheld by Newell (2005) who expresses that it is vital to have equipped faculty in associations, which is satisfied with a viable enlistment and determination measure.

On the off chance that some unacceptable individual is employed, the association can experience the ill effects of a few conservative misfortunes all things being equal (Newell, 2005; Muir 1988). Notwithstanding, having the option to recruit the most capable and best representatives available is getting progressively hard among the opposition hands on market (Taylor and Collins, 2000; O'Donovan, 2019). The manner in which enrollment is being directed has consequently, because of the opposition, changed. It is not, at this point conceivable to utilize a similar enlistment sources as in the past, rather organizations these days utilize more imaginative methods of selecting their representatives as an approach to stand apart from contenders (Taylor and Collins, 2000). What can be drawn from this is the manner by which significant it is for each association to attempt to stay aware of enlistment patterns and how enrollment is creating.

\section{Artificial Intelligence}

Artificial Intelligence (AI) has been around for quite a while and have had a wide space of utilization consistently, however just during the later year has the innovation been additionally evolved and executed inside various authoritative settings (Tecuci, 2012). To comprehend the idea of AI, the simplest path is to separate the words without help from anyone else to take a gander at each significance. In any case, despite the fact that AI have been around for a more extended timeframe there isn't one predecided meaning of the idea (Legg and Hutter, 2007). Numerous explores who present definitions center to characterize the 'I' in AI, for the most part since this is harder to pinpoint. The meaning of ' $\mathrm{A}$ ' is Artificial, generally concurred on term and subsequently doesn't require as much characterizing (Bringsjord and Schimanski, 2003). Artificial, characterized by Oxford Dictionary is something "made or created by people instead of happening normally, particularly as a duplicate of something characteristic" (Oxford Dictionary, 2019). Hence, it can be set up that artificial is the things that people have made to reenact something that generally happens normally.

The precarious part at that point exists in characterizing intelligence. Some would characterize the term of AI as the making of robots, machines or projects which possess what could be viewed as comparable clever conduct as human have (Tecuci, 2012; Kaplan, 2016). The issue with this definition is estimating human intelligence to contrast it with that of the robots or machines possessing it. Kaplan (2016) rather expresses that his very own understanding of intelligence would be that it is "the capacity to make suitable speculations in an ideal design dependent on restricted information" (p.5). Numerous other more casual meanings of intelligence incorporate it being when something can think, plan, have information, adjust to climate or recover data (Legg and Hutter, 2007).

It could likewise be the capacity to get information and from that settle on choices dependent on the information just as the current circumstance (Ved, Kaundanya and Panda, 2016). For instance, it very well may be that a program can figure out how to mess around, for example, spasm tac-toe, or how to perceive singular faces or make music - at that point it is artificial intelligence (Kaplan, 2016). With the end goal of this investigation, AI is characterized as the capacity of such things as machines to learn, decipher and comprehend all alone along these lines to that of people.

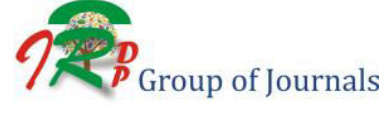




\section{The Application of Artificial Intelligence in Recruitment}

As indicated by Upadhyay and Khandelwal (2018), the utilization of AI in HRM was perhaps the most momentous patterns among enlistment experts in 2018. Stuart and Norvig (2016) characterizes data extraction as a cycle where data and information can be assembled by examining a book. Particularly in enrollment of new representatives, AI can be utilized by data extraction strategies that can make the cycle of resume examining and extraction of pertinent data computerized (Kaczmarek, Kowalkiewicz and Piskorski, 2005). Since the quantity of requests for employment have expanded and can even overpower HR divisions, mechanized frameworks that positions work competitors have been introduced to speed up the recruiting cycle. HR division for the most part physically lead the assessment of the got requests for employment, consequently candidate positioning frameworks which can be made with the use of AI can make enrollment specialists assessment task more productive (Faliagka, Ramantas, Tsakalidis and Tzimas, 2012). Competitor positioning framework works at the force of $\mathrm{AI}$ and human enrollment specialists gives information to the AI calculations, from where they gain proficiency with the scoring capacity of candidates (Faliagka et al. 2012).

Upadhyay and Khandelwal (2018) present chat bots that are AI-driven enrollment collaborators that empower individual and exceptional association prospects with competitors through messages, instant messages or exchange box. There are a few PC upheld work matchmaking methods which have been created to facilitate the responsibility of selection representatives. Such strategies incorporate programming that sorts continues and can be executed by abusing learning-based procedures and calculations (Montuschi, Gatteschi, Lamberti, Sanna, and Demartini, 2014).An intriguing element of AI-based positioning frameworks is the likelihood to assemble data about candidates' character characteristics that are critical when satisfying position positions. Notwithstanding, these characteristics are regularly seen during new employee screening, yet starter information can be obtained through web look. By directing phonetic investigation to candidates' blog entry or LinkedIn pages, it is feasible to accumulate data about candidates' character trails, state of mind and feelings (Faliagka et al. 2012). Prospective employee meetings led as a video meet have become a mainstream enrolling instrument among organizations. An application for video meets that use AI has been created by HireVue. In this application AI can decipher and dissect candidate's nonverbal communication, looks or manner of speaking. The application analyzes the talked with candidates to the top ability representatives in the organization lastly propose the best candidates to scouts (HireVue, 2018). The worldwide lodging network Hilton encountered a few advantages of directing video interviews, though the most striking ramifications was the decline in the measure of time spent in enrollment measure. Before the enlistment cycle took 42 says in Hilton lodging, however because of utilization of AI based video interviews, it requires just 5 days (HireVue Case Study, 2017).

\section{Advantages of Artificial Intelligence based Recruitment}

As indicated by Dickson and Nusair (2010) the point of enlistment frameworks is to ease associations and save use by modernizing their enrollment interaction. Enrollment frameworks are intended to make the enlistment cycle faster by various types of capacities, for example, pre-screening and arranging resumes and afterward coordinating with these resumes to open occupation opportunities. Consequently this improve supervisors' undertaking with regards to securing qualified position candidates both in the provisions of sped up and proficiency (Dickson and Nusair, 2010). Likewise, Dickson and Nusair (2010) tracked down that the utilization of AI in enrollment measure empowers associations to arrive at bigger applicant pool and there is less administrative work to be finished. Besides, AI can skim the information that is posted via online media and henceforth it is feasible to gain admittance to candidate's qualities, perspectives furthermore, character characteristics (Upadhyay and Khandelwal, 2018) that generally have been examined during the prospective employee meeting (Faliagka et al. 2012). Henceforth, because of AI frameworks it is feasible for enrollment specialists to check work candidates' character attributes as of now before a new employee screening (Faliagka et al. 2012). Upadhyay and Khandelwal (2018) specifies that AI go about as impartial and resumes are screened genuinely such that it gives equivalent opportunity to all candidates. With regards to up-and-comers who were dismissed from the work opportunity, AI frameworks permit input about their capabilities and abilities that these competitors can grow further later on (Upadhyay and Khandelwal, 2018).

By leading customary vis-à-vis interviews with potential occupation candidates, associations face a few expenses, including the expenses of chiefs and administrators who are available during the meeting and employing measures. Notwithstanding these advantages, the diminished measure of manual work in employing measure yield more opportunity to zero in on those potential occupation applicants who are reasonable for accessible occupation opening (Guchait, Ruetzler, Taylor and Toldi, 2013). As per Leong (2018), the utilization of AI in enrolling empowers spotters to interface with the best ability the board up-and-comers in a split second as 
opposed to investing gigantically energy and assets on perusing and looking over got resumes. Simulated intelligence based enrollment and ability choice empowers to rank occupation competitors and consequently to perceive the top-scoring up-and-comers. Leong (2013) call this interaction as Resume Scorer and his cycle save scouts time and exertion altogether. Notwithstanding these progressions, AI can help spotters with regards to conveying modified messages to conceivable occupation up-and-comers about the current status of their employment forms just as planning interviews. Upadhyay and Khandelwal (2018) brings up that already dull undertakings were directed by human enrollment specialists, however AI will make a portion of the enlistment measures outdated. This thusly permits scouts to designate the redundant errands to AI frameworks and subsequently selection representatives have more assets to put on essential issues (Upadhyay and Khandelwal, 2018). With regards to associating with competitors, it tends to be expressed that AI frameworks work with the correspondence among up-and-comers and enrollment specialists, since AI frameworks permits to contact up-andcomers through the web, social channels and versatile stages (Upadhyay and Khandelwal, 2018).

\section{Difficulties of Artificial Intelligence based Recruitment}

Indeed, even there are examines led expressing that new innovation and huge information makes HRM more effective and exact (Zang and Ye, 2015), there are individuals who consider that human asset investigation can be just a transient pattern if the innovation change doesn't figure out how to become a proceeding with part of the executives dynamic (Rasmussen and Ulrich, 2015). One astounding aggregate of difficulties that AI-based enlistment involves is close to home security and the way how information is taken care of and broke down. It concerns both HR experts and online HRM clients with regards to examining information or sharing own data (Bondarouk and Brewster, 2016). Martincevic and Kozina (2018) sees it practically outlandish for associations to work effectively with no amount of transformation of new innovations. The capacity to adjust new innovation in associations decides generally how they are capable accomplish their market intensity. Past examinations have shown that the transformation of new innovation involves a few advantages with regards to improved execution (Martincevic and Kozina, 2018). This proclamation portrays the adequacy such that measures which before required human astute and incorporated a few isolated and tedious parts, would now be able to be directed by AI instruments in a lot more limited time. Moreover, adequacy can suggest an abatement in the measure of time spent in enrollment measure. This kind of adequacy was inspected in the Hilton inn case model, where AI based video interviews had the option to chop down the enlistment interaction from 42 days to 5 days (HireVue Case Study, 2017.)

\section{Conclusion}

Thus it's clearly evident from the study by eminent Researchers that Artificial Intelligence plays a crucial role in extracting needed information from the pool of applicants but few difficulties are experienced when it comes to innovation in recent days. The creators contend that ability deficiencies are probably the biggest test in employing industry. This contention is in the line with one of the talked with experts concurred that AI may experience issues with regards to social contrasts: "Language predispositions and social comprehension of machine are likewise difficulties for AI. A region where AI may experience issues understanding social boundaries what wording is and how data is introduced in certain country or culture." Computer based intelligence is accepted to have the option to supplant regulatory assignments in both HRM yet additionally particularly inside the enrollment cycle in the enlistment exercises, mediating position candidate variable and the enlistment results. In these parts AI would stretch out on the conventional enlistment measure and have the option to give more broad options in contrast to both the organization and occupation candidates simultaneously it gets both quicker and smoother. By utilizing AI programming in the conventional enrollment measure an organization might actually get brings about their correspondence with competitors, bigger up-and-comer pool, re-revelation of lost gifts and generally speaking improved enlistment results. Be that as it may, the utilization of AI programming inside these spaces of the enlistment interaction should intently be inspected independently by each organization to check whether the manner in which $\mathrm{AI}$ is misused in enrollment is something which is a need for their organization.

\section{Reference}

[1] Braun, V., \& Clarke, V. (2006). Using thematic analysis in psychology. Qualitative Research in Psychology, 3, 77-101.

[2] Acikgoz, Y. (2019). "Employee recruitment and job search: Towards a multi-level integration". Human resource management review, 29, 1-13

[3] Bibi, P., Pangil, F., \& Johari, J. (2016). HRM practices and employees' retention: the perspective of job embeddedness theory. Asian Journal of multidisciplinary study, 4(5), 41-47

[4] Beattie, G. \& Johnson, P. (2012) Possible unconscious bias in recruitment and promotion and the need to promote equality. Perspectives: Policy and Practice in Higher Education, 16:1, 7-13

[5] Ambrose, M., \& Kulik, C. (1993). Old friends, new faces: motivation research in the 1990's Journal of management. 25(3), 231-292.

[6] Walsh, David. 2017. Employment Law for Human Resource Practice. Mason, Ohio: South-Western.

[7] Spielkamp, Michael. 2017. Inspecting Algorithms for Bias. MIT 
Technology Review. June 12. https://www.technologyreview.com/s /607955/inspecting-algorithms-for- bias/

[8] Pfeffer, Jeffrey and Robert I. Sutton. 2006. Hard Facts, Dangerous Half-Truths and Total Nonsense: Profiting from Evidence-Based Management. Harvard Business Review Press.

[9] Meyer, David. 2018. Amazon Reportedly Killed an AI Recruitment System Because It Couldn't Stop the Tool from Discriminating Against Women. Fortune. October 10th. http://fortune.com/ 2018/10/10/amazon-ai-recruitment-bias-women-sexist/

[10] Lind, E. Allan and Kees Van den Bos. 2002. "When Fairness Works: Toward a General Theory of Uncertainty Management.' Research in Organizational Behavior 24: 181-223.

[11] Dietvorst, B. J., Simmons, J. P., \& Massey, C. (2016). Overcoming algorithm aversion: People will use imperfect algorithms if they can (even slightly) modify them. Management Science, 64(3), 11551170.

[12] Adler, Paul and Bryan Boris. 1996. "Two Types of Bureaucracy: Enabling and Coercive." Administrative Science Quarterly 41: 6189.

[13] Pfeffer, Jeffrey and Robert I. Sutton. 2006. Hard Facts, Dangerous Half-Truths and Total Nonsense: Profiting from Evidence-Based Management. Harvard Business Review Press.

[14] Stone, Peter. 2011 The Luck of the Draw: The Role of Lotteries in Decision Making. Oxford: Oxford University Press.

[15] Rousseau, Denise (Editor). 2014. The Oxford Handbook of Evidence-Based Management. Oxford University Press. 